\title{
Combined Carbon Dioxide Laser Lateral Canthotomy and Femtosecond Laser-Assisted Cataract Surgery ${ }^{*}$
}

\author{
Randal Tanh Hoang Pham ${ }^{1,2}$ \\ ${ }^{1}$ Aesthetic and Refractive Surgery Medical Center, San Jose, USA; ${ }^{2}$ Advanced Surgery Medical Center, San Jose, USA. \\ Email: randalpham@lasernews.com
}

Received September $10^{\text {th }}, 2013$; revised October $6^{\text {th }}, 2013$; accepted October $13^{\text {th }}, 2013$

Copyright (C) 2013 Randal Tanh Hoang Pham. This is an open access article distributed under the Creative Commons Attribution License, which permits unrestricted use, distribution, and reproduction in any medium, provided the original work is properly cited.

\begin{abstract}
Purpose: The purpose of this study was to evaluate a new procedure aimed at lengthening the palpebral fissure to facilitate femtosecond laser-assisted cataract surgery in patients with small eyes. Method: A quick procedure using the $\mathrm{CO}_{2}$ laser was adapted for patients with small eyes undergoing laser-assisted cataract surgery using the Catalys system. The UniPulse $\mathrm{CO}_{2}$ laser (Nidek) was used for laser lateral canthotomy on patients with small palpebral fissures to allow fitting of the Liquid Optic ${ }^{\mathrm{TM}}$ Interface eyepiece. Results: Lateral canthotomies were performed on 19 women and 7 men (ages ranged from 45 to 93 years) with lower eyelid lengths equal to or shorter than $32 \mathrm{~mm}$ who then underwent femtosecond laser-assisted cataract surgery. A total of 33 eyelids received laser lateral canthotomy with the $\mathrm{CO}_{2}$ laser; only one eyelid had lateral canthotomy with cold-steel tenotomy scissors. Dockings were completed for all 34 eyes with eyelids receiving lateral canthotomies. The 33 eyes with eyelids that received laser canthotomy with the $\mathrm{CO}_{2}$ laser had successful femtosecond laser-assisted cataract surgery. The one eye with the eyelid that received cold-steel canthotomy could not have femtosecond laser-assisted cataract surgery despite successful docking because of pupillary constriction. The findings were statistically significant; Fisher Exact Test showed a p-value of 0.0294. Conclusion: Laser lateral canthotomy with $\mathrm{CO}_{2}$ laser is a safe and effective method to allow docking and completion of femtosecond laser-assisted cataract surgery.
\end{abstract}

Keywords: Cataract; Laser; $\mathrm{CO}_{2}$ Laser; Femtosecond Laser; Femtosecond Laser-Assisted Cataract Surgery; Refractive Surgery; Laser Refractive surgery; LASIK; PRK; LASEK; ALK; RLE; EpiLASIK; PRELEX; ICR; Phakic Intraocular Lens Implant; AK; RK; Cataract Surgery San Jose

\section{Introduction}

Femtosecond laser-assisted cataract surgery (FLACS) has recently been available in the United States and around the world. The advantages of this technology include the ability to create a perfectly sized, shaped and centered circular anterior capsular incision, the perfect positioning of the intraocular lens held by a complete edge of the anterior capsule and the ability to break up the cataract, which in turn reduces phacoemulsification time and energy $[1,2]$. During the procedure, a suction ring is placed directly on the eyeball and the laser is attached to the ring using suction; this interface stabilizes the eye relative to the laser. Several factors can affect the ability to create a stable laser-eye interface using the suction ring. In cases where the eye has a short palpebral fissure i.e. short lower eyelid length (LEL), fitting the

"Financial Disclosure: The author has no financial disclosure or conflict of interest. suction ring to the eyeball may be difficult. In addition, suction can be lost with excessive eye, head and/or body movements [3]. This retrospective study was conducted to identify eyelids that required lateral canthotomy due to a short palpebral fissure, factors that were responsible for femtosecond laser docking failure and to evaluate the safety and efficacy of laser lateral canthotomy (LLC) as an adjunct procedure to femtosecond laser-assisted cataract surgery. A review of the literature showed that this study was the first of its kind to compare cold-steel and laser lateral canthotomies when combined with laserassisted cataract surgery and to address issues related to docking and completion of the femtosecond laser-assisted cataract surgery.

\section{Materials and Methods}

Charts were reviewed for all patients who had undergone femtosecond laser-assisted cataract surgery with the 
Catalys $^{\mathrm{TM}}$ Precision Laser System (Optimedica, Santa Clara, CA, USA) combined with either cold-steel or laser lateral canthotomy with the Nidek Unipulse $\mathrm{CO}_{2}$ laser (Nidek, Fremont, CA, USA) between September 2012 and July 2013. Demographic data (age, sex, race), use of anticoagulants, indications for lateral canthotomy (exposure resistant factors [ERF's]), and occurrence of postoperative complications (infection, bleeding, non-healing and scarring of lateral canthus, lower eyelid ectropion and formation of conjunctival cysts and cataract surgery complications i.e. ruptured anterior or posterior capsules, dropped nucleus intraoperatively or hypotony, shallow/ flat anterior chamber, distorted pupil, intraocular lens dislocation, vitreous herniation, loss of nuclear or cortical materials into the vitreous, retinal detachment and endophthalmitis) were noted for each patient. The minimum lower eyelid length required (MR LEL) for femtosecond laser docking with patient interface-Liquid Optic ${ }^{\mathrm{TM}}$ Interface (LOI) (Optimedica, Santa Clara, CA, USA) was also determined. Cold-steel and laser lateral canthotomies were compared with respect to successful completion of femtosecond laser-assisted cataract surgery. Statistical significance was assessed using the two-tailed Fisher Exact Test.

\section{Surgical Technique}

The patient was placed on the Catalys ${ }^{\mathrm{TM}}$ Precision Laser System operating table (Optimedica, Santa Clara, CA, USA). The Liquid Optic ${ }^{\text {TM }}$ Interface (Optimedica, Santa Clara, CA, USA) was fitted on the eye. If the Liquid Optic $^{\mathrm{TM}}$ Interface could not be fitted or successful docking could not be achieved, then the patient was prepared for lateral canthotomy. Successful docking was defined as achieving a suction level accepted by the Catalys ${ }^{\text {TM }}$ Precision Laser System and maintained throughout the procedure. The lower eyelid length was measured and marked with a fine tip Devon ${ }^{\mathrm{TM}}$ marking pen (Covidien, Mansfield, MA, USA). A photograph of the lateral canthus of the operative eye was taken using the Nikon 7100D camera (Nikon, Melville, NY, USA). Application of the topical anesthetic EMLA cream (APP, Lake Zurich, IL, USA) to the lateral canthus of the operative eye followed by injection of $0.5 \mathrm{cc}$ of $2 \%$ Lidocaine with 1:100,000 epinephrine local anesthetic solution (Hospira, Lake Forest, IL, USA) into the lateral canthus of the operative eye was performed. The patient's lateral canthus of the operative eye was prepped with $5 \%$ Betadine solution. For non-laser lateral canthotomy a hemostat was placed over the lateral canthus for 5 minutes to control hemostasis. Tenotomy scissors were used to make an incision into the lateral canthal commissure to achieve the minimum lower eyelid length required for femtosecond laser docking of the interface eyepiece. Pressure was applied to the lateral canthus to control hemostasis. For laser lateral canthotomy a non-reflective metal forceps (Oculoplastik, Montreal, Quebec, Canada) was used to protect the eye during laser lateral canthotomy. The lateral canthal commissure was incised with the Nidek Unipulse $\mathrm{CO}_{2}$ laser set at 5 watts in Unipulse mode level III (mid-level between coagulation and cutting modes) to achieve the minimum lower eyelid length required for femtosecond laser docking. For laser lateral canthotomy pressure to the lateral canthus was not performed. For both cold-steel and laser lateral canthotomies no wound closure was performed. FLACS was then performed on all patients starting with the fitting of the LOI. The details of FLACS technique was previously described $[1,2]$.

\section{Results}

An adequate exposure for fitting and successful femtosecond laser docking with the Liquid Optic ${ }^{\mathrm{TM}}$ Interface required a minimum lower eyelid length of $32 \mathrm{~mm}$. Thirty-four eyelids (from 26 patients) were identified to receive lateral canthotomy because of fitting failure or loss of suction; eight patients had bilateral combined lateral canthotomy and cataract surgery performed on different days. The patient ages ranged from 45 to 93 years. Nineteen patients were female and seven were male. Twenty-two were Asians and four were Caucasians. Six patients were on anticoagulants (two on warfarin, four on aspirin). Of these six patients, seven eyelids had lateral canthotomy ( 1 eyelid with cold-steel and 6 with laser). Post-operative follow up for all patients ranged from 3 to 12 months.

The following exposure resistant factors were identified: small palpebral fissure (32 eyelids), excessive squeezing ( 1 eyelid), excessive eye movements-nystagmus ( 2 eyes), excessive body movements ( 1 eye), abnormal eyelid-dermatochalasis (23 eyelids), entropion (1 eyelid), and abnormal conjunctiva-pingueculum (2 eyes).

No infection, non-healing or scarring of lateral canthal wound, conjunctival cysts, or ectropion was noted in this study (Figures 1-4). One case of lateral canthal bleeding occurred after cold-steel lateral canthotomy in a 93 yearold Asian female patient who was taking anticoagulant (warfarin) at the time of surgery. Docking was successful in this patient but femtosecond laser procedure was not completed because of pupillary constriction after several docking attempts. Conventional cataract surgery, however, was performed. When this patient underwent cataract surgery for the second eye laser lateral canthotomy was performed; no canthal bleeding was noted and femtosecond laser-assisted cataract surgery was completed without complication. Comparison of cold-steel versus laser lateral canthotomy showed that all eyes that had laser lateral canthotomy had completion of femtosecond laser procedure. Two-tailed Fisher Exact Test showed a p-value of 0.0294 . No complication either from conven- 


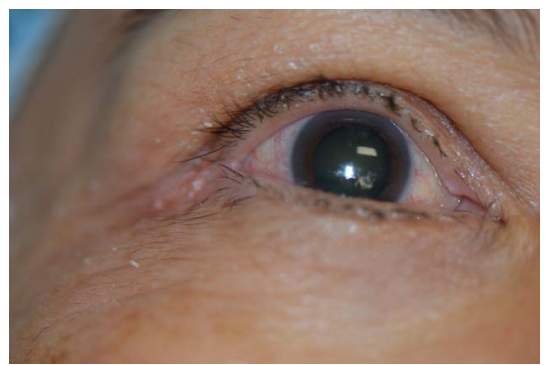

Figure 1. Pre-op photograph of right eye with cataract and intact lateral canthus.

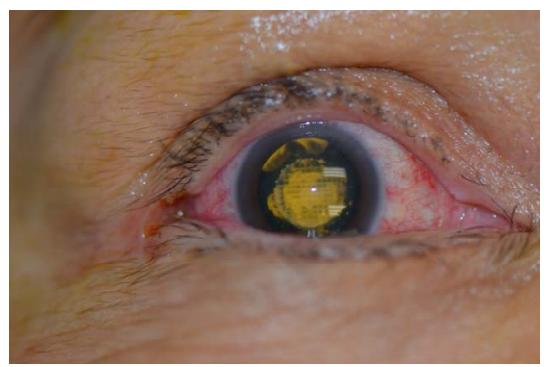

Figure 2. Immediate post-op photograph of right eye after combined LLC and FLACS. Photograph showed complete hemostasis of lateral canthus with no suture placed.

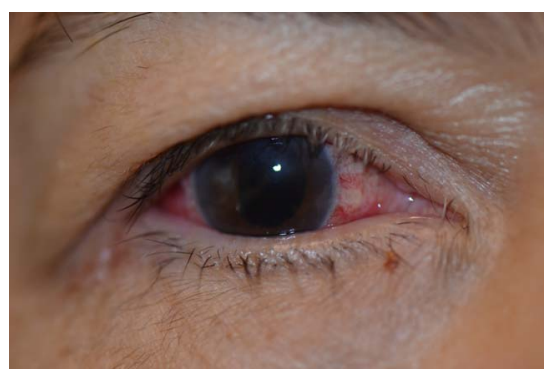

Figure 3. Day 1 post-op photograph of right eye after combined LLC and FLACS. Photograph showed complete lateral canthal wound apposition.

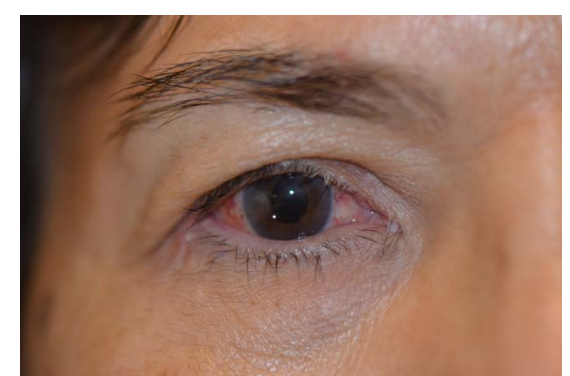

Figure 4. Day 5 post-op photograph of right eye showed complete wound healing of lateral canthus.

tional cataract surgery or from FLACS was identified.

\section{Discussion}

Patients undergoing femtosecond laser-assisted cataract surgery required a minimum lower eyelid length of $32 \mathrm{~mm}$ in order to fit the Liquid Optic ${ }^{\mathrm{TM}}$ Interface over the eyeball, thus ensuring adequate exposure of the eye to the laser beam. It was noted, however, that the circumference of the LOI provided adequate stabilization once the interface was fitted on the patients' eyes. This stabilization could not be achieved with interfaces that had circumferences smaller than $32 \mathrm{~mm}$. Lack of stabilization could cause loss of suction during femtosecond laser procedure. Stabilization was also affected by the ERF's. The ERF's identified in this study fell into three categories: inadequate exposure, irregular conjunctival surface and excessive eye and/or body movements. All ERF's for each patient should be identified prior to surgery and these findings should be incorporated into the pre-operative plan. ERF's and lateral canthotomy should be discussed with patients pre-operatively and inform consent obtained. This approach will ease the patient's anxiety and provide the surgeon with a well-defined protocol to follow on the day of surgery. A flowchart is included to assist in the decision making process prior to surgery (Figure 5). This flowchart can be applied to other patient interfaces using the following formula:

Interface Circumference/2 (in mm) = Minimum Required Lower Eyelid Length (MR LEL) for interface (in mm).

Carbon dioxide laser was found to provide superior hemostasis when compared to cold-steel [4]. A previous study also showed that use of the $\mathrm{CO}_{2}$ laser in the lateral canthal area was safe and effective [5]. The only patient in the present study who developed lateral canthal bleeding had cold-steel canthotomy. This patient, who was the author's first patient to receive FLACS, required additional time for pre- and post-canthotomy hemostasis and multiple attempts were needed to achieve adequate suction for placement of the Liquid Optic ${ }^{\mathrm{TM}}$ Interface. Pupillary constriction occurred in this patient and the femtosecond laser-assisted procedure could not be completed. When the same patient underwent cataract surgery for the second eye laser lateral canthotomy was performed and completion of femtosecond laser procedure was achieved. This case illustrates the importance of hemo-

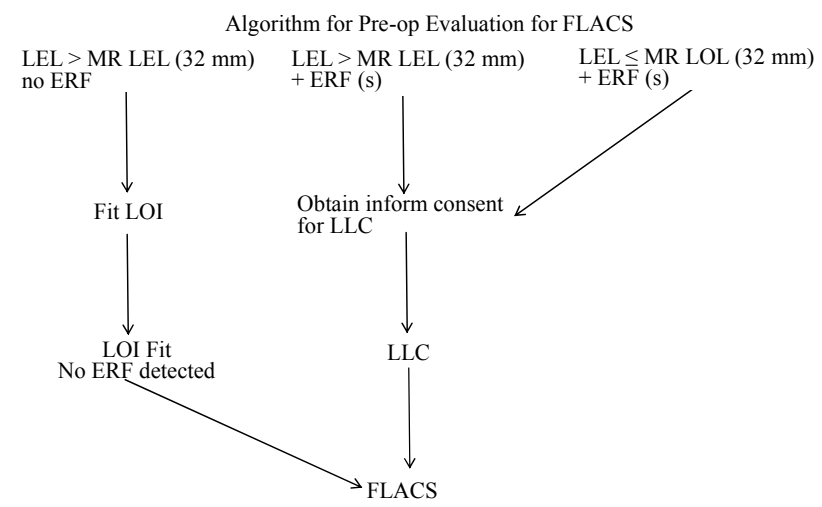

Figure 5. Flowchart for pre-op evaluation for FLACS. 
stasis control should lateral canthotomy become indicated for femtosecond laser-assisted cataract surgery. This patient was one of the six patients in the study who received anticoagulants and one of two who was on warfarin. None of the patients undergoing $\mathrm{CO}_{2}$ laser lateral canthotomy developed canthal bleeding. This observation reinforces the finding that $\mathrm{CO}_{2}$ laser lateral canthotomy provides superior hemostasis compared to coldsteel and is therefore indicated in patients who receive anticoagulants [4]. Continued use of anticoagulants is currently considered the standard of care for patients undergoing cataract surgery in many communities in North America, Europe, and Japan [6-8].

$\mathrm{CO}_{2}$ laser lateral canthotomy can be used as an adjunct procedure for conventional cataract surgery and other refractive surgeries such as LASIK, LASEK, PRK, ALK, RLE, EpiLASIK, PRELEX, ICR, phakic intraocular lens implant, AK, RK, etc. In addition, in patients with multiple ERF's, $\mathrm{CO}_{2}$ laser lateral canthotomy can be effective in reducing incidences of suction loss during femtosecond laser refractive surgery. This advantage helps achieve successful completion of femtosecond laser-assisted procedures.

\section{Conclusion}

$\mathrm{CO}_{2}$ laser lateral canthotomy is a safe and effective adjunct procedure for femtosecond laser-assisted cataract surgery.

\section{Acknowledgements}

The author wishes to thank Beverley Murray, $\mathrm{PhD}$ for her assistance in the preparation of this manuscript.

\section{REFERENCES}

[1] I. Conrad-Hengerer, et al., "Effects of Femtosecond Laser Fragmentation of the Nucleus with Different Softening
Grid Sizes on Effective Phaco Time in Cataract Surgery," Journal of Cataract \& Refractive Surgery, Vol. 38, No. 110, 2012, pp. 1888-1894. http://dx.doi.org/10.1016/j.jcrs.2012.07.023

[2] R. G. Abell, et al., "Catalys Femtosecond Laser-Assisted Cataract Surgery Compared to Conventional Cataract Surgery," Clinical \& Experimental Ophthalmology, Vol. 41, No. 5, 2013, pp. 455-462. http://dx.doi.org/10.1111/ceo.12025

[3] R. Pham, "Lateral Canthotomy for Small Eyes Undergoing Laser Refractive Cataract Surgery with Image Guidance System," Presented at the 2013 Annual Symposium of the American Society for Cataract and Refractive Surgery, San Francisco.

[4] A. M. Goldbaum, et al., "The $\mathrm{CO}_{2}$ Laser in Oculoplastic Surgery," Survey of Ophthalmology, Vol. 42, No. 3, 1997. pp. 255-267. http://dx.doi.org/10.1016/S0039-6257(97)00097-0

[5] S. S. Baker, et al., "Lateral Canthal Tendon Suspension Using the $\mathrm{CO}_{2}$ Laser: A Modified Technique," Dermatologic Surgery, Vol. 21, No. 12, 1995, pp. 1071-1073. http://dx.doi.org/10.1111/j.1524-4725.1995.tb00561.x

[6] I. S. Barequet, et al., "Phacoemulsification of Cataract in Patients Receiving Coumadin Therapy: Ocular and Hematologic Risk Assessment," American Journal of Ophthalmology, Vol. 144, No. 5, 2007, pp. 719-723. http://dx.doi.org/10.1016/j.ajo.2007.07.029

[7] R. Batra, et al., "Perioperative Management of Anticoagulated Patients Having Cataract Surgery," Journal of Cataract \& Refractive Surgery, Vol. 35, No. 10, 2009, pp. 1815-1820. http://dx.doi.org/10.1016/j.jcrs.2009.05.042

[8] H. Kobayashi, "Evaluation of the Need to Discontinue Antiplatelet and Anticoagulant Medications before Cataract Surgery," Journal of Cataract \& Refractive Surgery, Vol. 36, No. 7, 2010, pp. 1115-1119. http://dx.doi.org/10.1016/j.jcrs.2010.01.017

\author{
Abbreviations \\ LEL: Lower Eyelid Length \\ MR LEL: Minimum Required Lower Eyelid Length \\ ERF: Exposure Resistant Factor \\ LOI: Liquid Optic ${ }^{\mathrm{TM}}$ Interface \\ LLC: Laser Lateral Canthotomy \\ FLACS: Femtosecond Laser-Assisted Cataract Surgery
}

